\title{
Benign pneumatosis intestinalis: a cause of massive pneumoperitoneum in the adult
}

\author{
David M. Liu, MD; William C. Torreggiani, MB, FRCR; Kevin Rowan, MD, BSc; \\ Savvas Nicolaou, MD
}

\begin{abstract}
Pneumatosis intestinalis (gas in the bowel wall) is often a benign condition, but it may mimic bowel ischemia or infarction and lead to unnecessary surgical intervention, especially when associated with pneumoperitoneum. We present a case of benign pneumatosis intestinalis with massive pneumoperitoneum and discuss various distinguishing features that may aid in its diagnosis.
\end{abstract}

Key words: pneumatosis intestinalis; radiography

\section{RÉSUMÉ}

La pneumatose kystique intestinale (présence de kystes gazeux dans la paroi intestinale) est souvent une affection bénigne mais qui peut être méprise pour une ischémie ou un infarctus intestinal et mener à une intervention chirurgicale inutile, surtout en association avec un pneumopéritoine. Nous présentons un cas de pneumatose bénigne accompagnée d'un pneumopéritoine massif et discutons des diverses caractéristiques distinctives qui pourraient en faciliter le diagnostic.

\section{Introduction}

The identification of pneumoperitoneum with intramural bowel gas is a cause for immediate attention. While the condition is often benign, up to $27 \%$ of patients with benign forms of pneumatosis may be misdiagnosed with surgical abdomens, and many of these proceed to unnecessary surgical intervention. ${ }^{1}$ Determination that the condition is benign and requires only conservative management depends on assessment of the clinical history, laboratory investigations and radiographic findings. We present a case of benign pneumoperitoneum and pneumatosis intestinalis secondary to chronic obstructive pulmonary disease (COPD).

\section{Case report}

A 68-year-old male presented to the emergency department with a history of sudden onset abdominal pain and distension that had commenced 3 hours prior to presentation. A background history of COPD was noted in addition to a history of myocardial infarction 5 years previously that required pacemaker insertion secondary to heart block. He had been otherwise well and had no previous history of gastrointestinal problems.

On examination, the abdomen was grossly distended, hypertympanic and mildly tender to deep palpation. Chest examination revealed bilateral expiratory wheezes and chest hyperinflation in keeping with COPD. Routine labo-

From the University of British Columbia, Vancouver, BC, and the Department of Radiology, Vancouver General Hospital, Vancouver, BC.

Received: May 20, 2003; final submission: Aug. 15, 2003; accepted: Sept. 15, 2003

This article has been peer reviewed.

Can J Emerg Med 2003;5(6):416-20 
ratory tests, including serum blood count, electrolytes and liver biochemistry were all within normal limits. Blood gases, cardiac enzymes, lactate dehydrogenase and serum lactate were normal. A provisional diagnosis of bowel obstruction was made, and plain chest and abdominal radiographs were obtained.

The erect chest radiograph (Fig. 1) revealed a large amount of free air under both hemidiaphragms, in keeping with massive pneumoperitoneum. Background changes of hyperinflation and distorted lung architecture were also present, reflecting the patient's known COPD. Plain abdominal films (Fig. 2) showed massive pneumoperitonuem and a large amount of intramural gas within the large and small bowel. Abdominal computed tomography (CT) was obtained to further investigate the suspected bowel obstruction.

The CT showed a large number of small gas-filled cysts arising from the wall of the small intestine, with lesser involvement of the colon, confirming pneumatosis intestinalis (Figs. 3a-3d). No abnormalities were identified in the arterial, venous or portal systems. Re-examination of the abdomen and a normal serum lactate suggested a benign process. A diagnosis of benign type pneumatosis intestinalis with secondary pneumoperitoneum was made based on the patient's clinical, laboratory and radiological findings.

The patient was admitted for treatment of the acute COPD exacerbation and discharged after 4 days. One

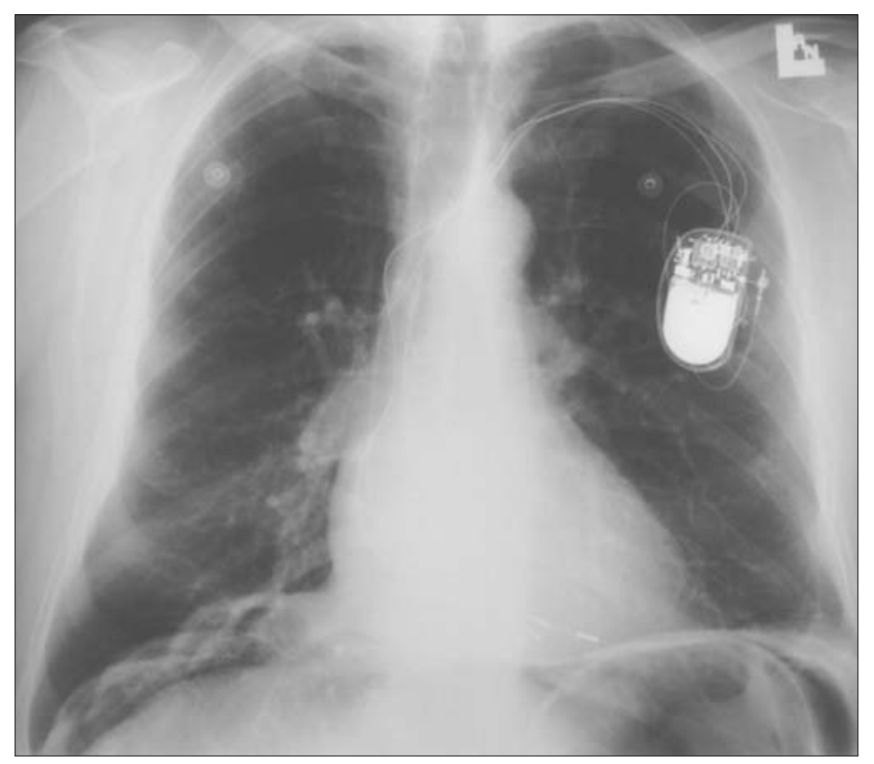

Fig. 1. Erect chest radiograph. Large air collections under the diaphragms in keeping with pneumoperitoneum. Associated findings of hyperinflation and lung parenchymal distortion compatible with history of chronic obstructive pulmonary disease. Incidental note of a dual lead pacemaker. month later, he again presented to the emergency department and had an abdominal CT for unrelated reasons. This CT scan demonstrated complete resolution of the process with no evidence of pneumoperitoneum, intramural bowel gas, or obstruction (Figs. 4a and 4b).

\section{Discussion}

The first description of pneumatosis intestinalis was by DuVeroni in $1730 .^{2}$ Pneumatosis was first defined as the presence of gas in an abnormal location in the body. This definition referred not only to the innocuous form, but also to more severe cases involving mucosal disruption and ischemic bowel necrosis. ${ }^{3}$ Synonymous terms for this condition include pneumatosis intestinalis coli, pneumatosis cystoides intestinorum, pneumatosis intestinalis cystoides and pneumatosis intestinorium cystoided hominus, ${ }^{4}$ as well as the clearest descriptor of all: benign intramural gas.

The case described here illustrates the fact that intramural air should be perceived not as a diagnosis, but rather a radiological finding. In fact, a number of conditions are associated with intramural gas (Table 1), and given the ambiguous nomenclature, the term pneumatosis intestinalis should be reserved for instances of benign intramural gas. This case also illustrates that clinical presentation, physical examination and laboratory results - not radiographic findings alone - should be considered when determining the cause and significance of intramural bowel gas.

\section{Pathophysiology and radiographic correlates}

When assessing the radiographic appearance of intramural gas, the organization and distribution of the gas is diagnos-

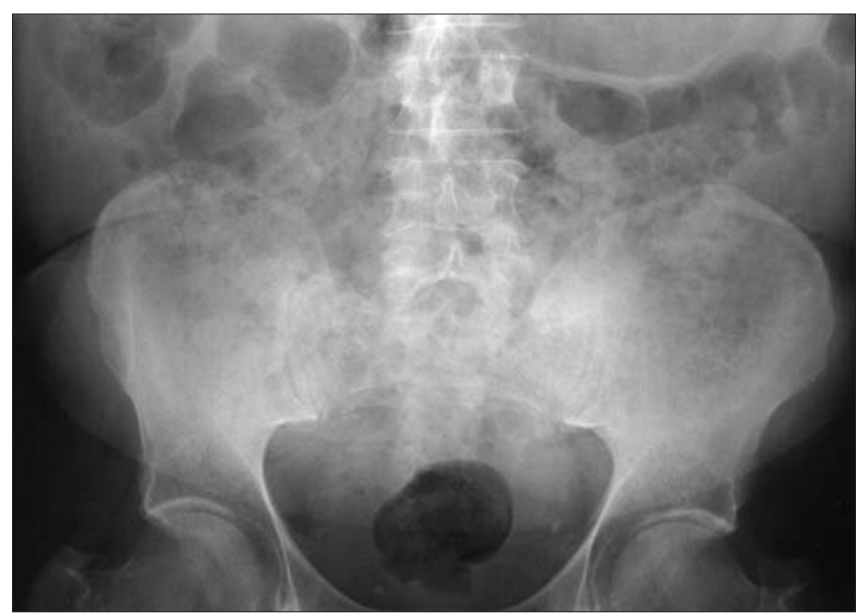

Fig. 2. Plain film abdominal series. Coned pelvis. Discrete loculated air-filled structures originating from the intramural portions of the small and large bowel are noted. The bowel gas distribution pattern is otherwise normal. 
tically helpful. As outlined by Heng and colleagues, typical patterns of intramural gas include microvesicular, cystic and linear variants. ${ }^{5}$

Microvesicular pneumatosis is typically associated with invasive procedures and is generally clinically and radiographically occult. Characterized by small collections of air within the lamina propria, it is a histological diagnosis, as the collections rarely exceed 100 micrometres in diameter. ${ }^{5}$ Due to its microscopic appearances on normal hematoxylin and eosin stains, it has also been coined mucosal pseudolipomatosis ${ }^{6}$ and is thought to result from microtrauma, however may also be related to the oxidation of residual hydrogen peroxide, left behind during sterilization of the instrumentation. ${ }^{7}$ A low-grade inflammatory process has been identified with this process, which may be sec- ondary to the introduction of air into the subserosal layers. ${ }^{8}$

The cystic (cystoids) variant of intramural gas is characterized by macroscopic submucosal cysts, ranging in size from a few millimetres to several centimetres in diameter. These protrude into the bowel lumen, creating characteristic indentations that can be seen radiographically and endoscopically. A number of etiologies have been associated with this condition, including COPD, immunosupression therapy, immunocompromise, steroid usage, inflammatory bowel disease and post bone marrow transplantation. ${ }^{2-3}$ Histologically, flattened macrophages line the cysts and multinucleated giant cells are commonly found, all suggestive of an inflammatory process although the cysts are always free of pathogens. ${ }^{5,9}$ Some studies suggest that the cysts are primarily air filled, ${ }^{10}$ supporting a mechanical the-
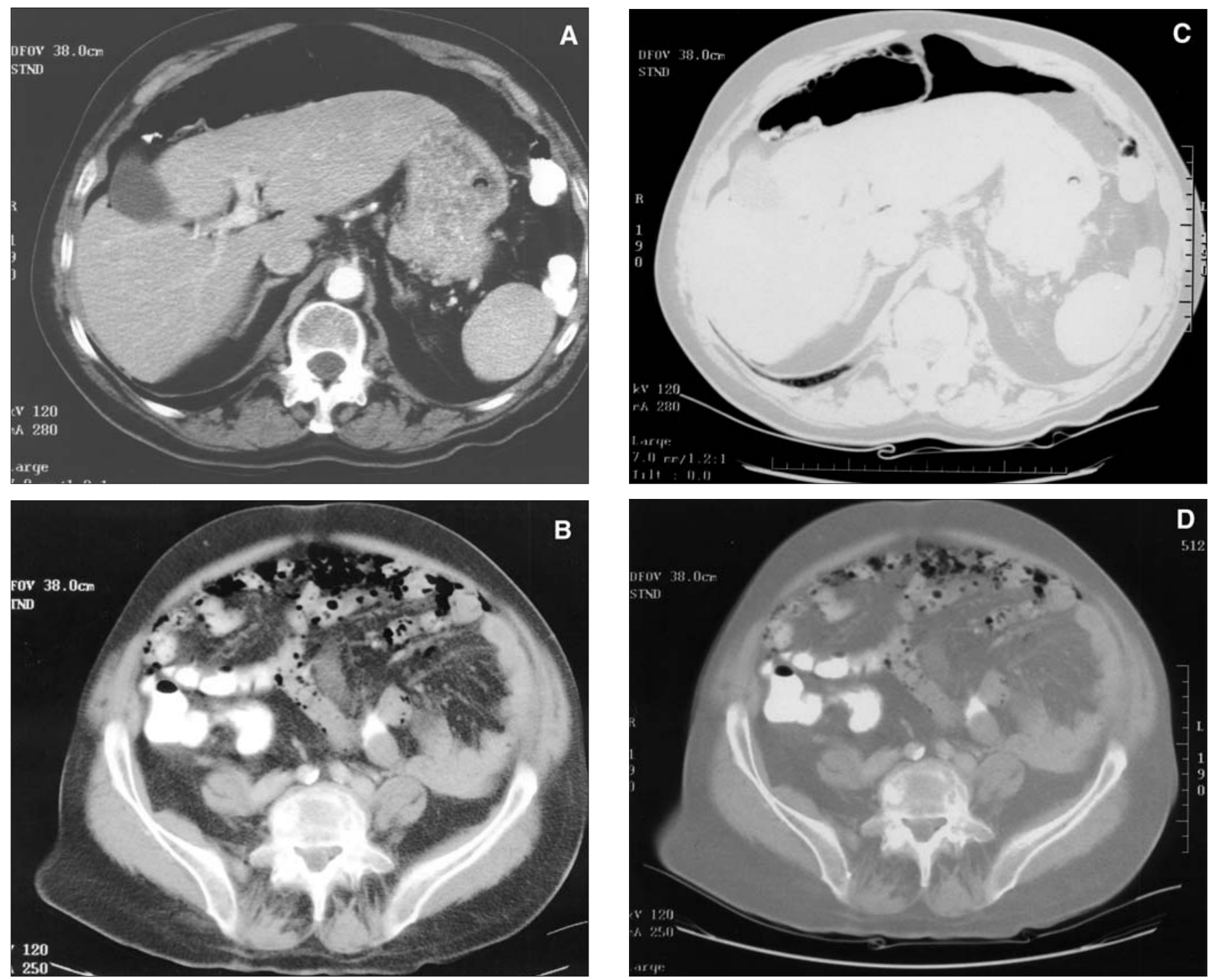

Fig. 3. Intravenous contrast enhanced CT of the abdomen with soft tissue and lung windows. Soft-tissue windows demonstrate normal appearing portal venous system and pneumoperitoneum (A) with air filled cystic structures clearly arising from small bowel (B). Lung windows show the pneumoperitoneum (C) and cyst-like intramural gas collections to better effect (D). 
ory of gas formation, while other studies have demonstrated high hydrogen concentrations, suggesting a bacterial origin for the gas. ${ }^{5}$ Regardless of the origin of the gas, it is clear that cyst rupture may produce a sterile pneumoperitoneum without peritonitis. ${ }^{4}$ Free intraperitoneal air is evident in up to $10 \%$ of patients with small bowel pneumatosis and $2 \%$ of those with large bowel pneumatosis. ${ }^{1}$

Some authors propose that, in patients with underlying lung disease, elevated intrathoracic pressures and pulmonary alveolar rupture lead to dissection of air along mediastinal blood vessels, through the diaphragm to major mesenteric vessels, ultimately collecting in the subserosal layer of the bowel wall. ${ }^{11}$ The cystic variant of intramural gas is nonspecific but suggestive of a benign etiology, par- ticularly if the clinical presentation supports this.

Linear pneumatosis carries a more ominous prognosis, as it is thought to represent the tracking of gas through compromised submucosa. This variant is identified by a streaking, linear or bubbly pattern that is oriented parallel to the bowel wall, plicae circulares or haustra. Although it can be associated with benign etiologies, linear intramural air suggests the need to rule out bowel ischemia or infarction, and mandates a search for portal venous gas - best detected using CT or ultrasound. Portal gas is an important prognostic feature, and if associated with bowel ischemia, mortality rates are as high as $83 \% .^{12}$

Necrotizing enterocolitis (NEC) is also associated with pneumatosis, but because $90 \%$ of cases present within the

\begin{tabular}{|c|c|c|}
\hline Category & Etiology & Configuration \\
\hline \multirow[t]{5}{*}{$\begin{array}{l}\text { Ischemia or } \\
\text { inflammation }\end{array}$} & $\begin{array}{l}\text { Toxic megacolon, pseudomembran- } \\
\text { ous colitis, irritable bowel disease }\end{array}$ & Linear \\
\hline & Bowel ischemia & Linear \\
\hline & Necrotizing enterocolitis & Linear \\
\hline & Volvulous / Obstruction & Linear / Cystic \\
\hline & Perforated diverticulum & Linear / Cystic \\
\hline \multirow{7}{*}{$\begin{array}{l}\text { Increased } \\
\text { intraluminal } \\
\text { pressure }\end{array}$} & $\begin{array}{l}\text { Chronic obstructive pulmonary } \\
\text { disease }\end{array}$ & Cystic \\
\hline & Asthma & Cystic \\
\hline & Positive pressure ventilation & Cystic \\
\hline & Pneumothorax / Mediastinum & Cystic \\
\hline & Post procedural (endoscopy, surgery) & Cystic \\
\hline & Cystic fibrosis & Cystic \\
\hline & Blunt abdominal trauma & Cystic \\
\hline
\end{tabular}
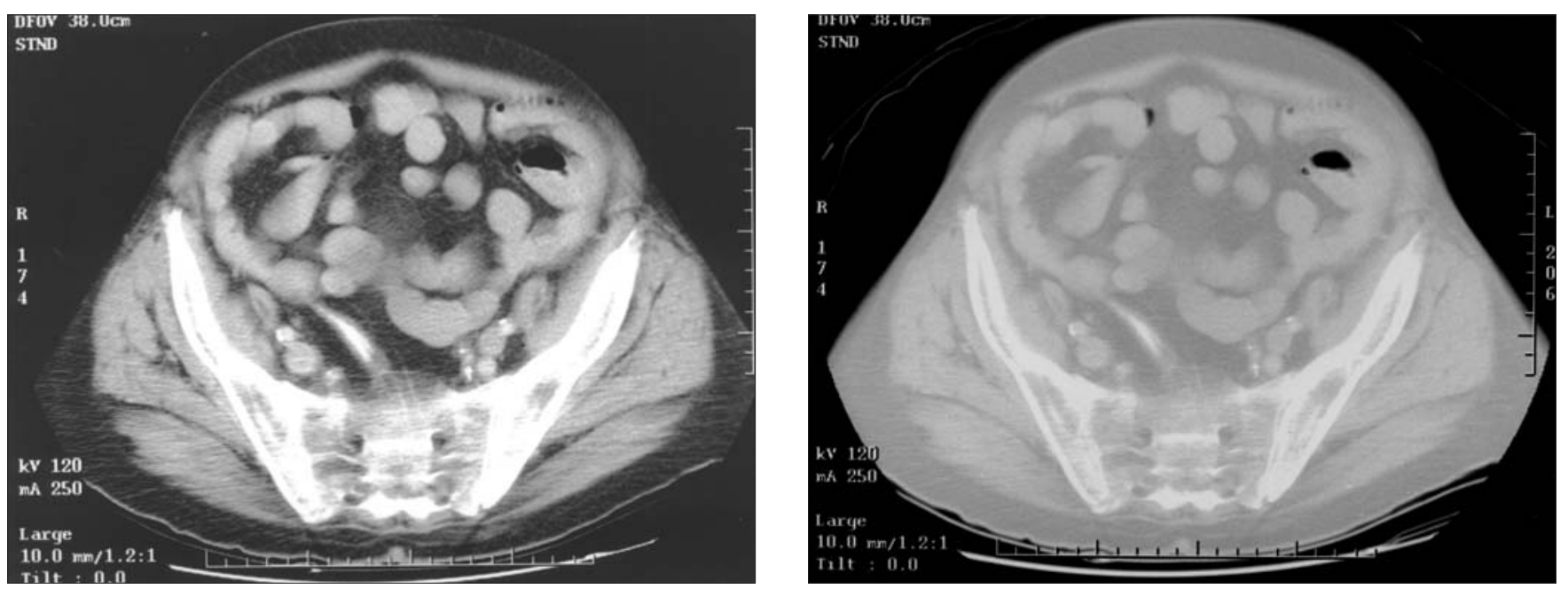

Fig. 4. Intravenous and oral contrast enhanced studies in the same patient 1 month later, demonstrated in soft tissue (A: left) and lung (B: right) windows. There is no further evidence of pneumatosis intestinalis, supporting the initial diagnosis of benign intramural gas. 
first 10 days of life, mostly in premature neonates, NEC is rarely a consideration in the adult population.

\section{Conclusion}

Pneumatosis intestinalis, air in the bowel wall, is a radiological or histological finding and is not a diagnosis in itself. As illustrated by the case presented, it is often a benign condition requiring only conservative treatment. Correct diagnosis and management is based on the results of clinical assessment, laboratory testing and imaging modalities. An understanding of the causes and associations of this finding will help physicians make appropriate decisions about surgical, medical or conservative management.

Competing interests: None declared.

\section{References}

1. Jamart J. Pneumatosis cystoides intestinalis. A statistical study of 919 cases. Acta Hepato Gastroenerol 1979; 26:419-22.

2. Holt S, Stewart I, Heading R. Resolution of primary pneumatosis coli. J R Coll Surg 1979;23:297-9.
3. Pear BL. Pneumatosis intestinalis: a review. Radiology 1998; 207(1):13-9.

4. Koss GL. Abdominal gas cysts (pneumatosis intestinorium cystoided hominus). Arch Path 1952;53:523-49.

5. Heng Y, Schuggler MD, Haggitt RD, Rohrmann CA. Pneumatosis intestinalis: a review. Am J Gastro 1995;90(10):1747-58.

6. Snover D, Sandstad J, Hutton S. Mucosal pseudolipomatosis of the colon. A J Clin Pathol 1985:84:575-80.

7. Knechtle SJ, Davidoff AM, Rice RP. Pneumatosis intestinalis: surgical management and clinical outcome. Ann Surg 1990; 212:160-5.

8. Pieterse AS, Leoung AS, Rowland R. The mucosal changes and pathogenosis of pneumatosis intestinalis cystoides intestinalis. Hum Pathol 1985;16:683-8.

9. Daly BD, Guthrie JA, Couse NF. Pneumoperitoneum without peritonitis. Postgrad Med J 1991;677:999-1003.

10. Kennedy J. Pneumatosis intestinalis. Clin Radiolo 1963;14:70-6.

11. Keyting W, McCarver R, Kovarik J. Pneumatosis intestinalis: a new concept. Radiology 1961;76:733-41.

12. Seaman W, Fleming R, Baker D. Pneumatosis intestinalis of the small bowel. Semin Roentgenol 1966;145:283-4.

Correspondence to: Dr. David M. Liu, Department of Radiology, JP Pavillion, Rm. 3550, Vancouver Hospital and Health Sciences Centre, 899 West 12th Ave., Vancouver BC V5Z 1M9; 604 839-3548, liudave@sbcglobal.net 